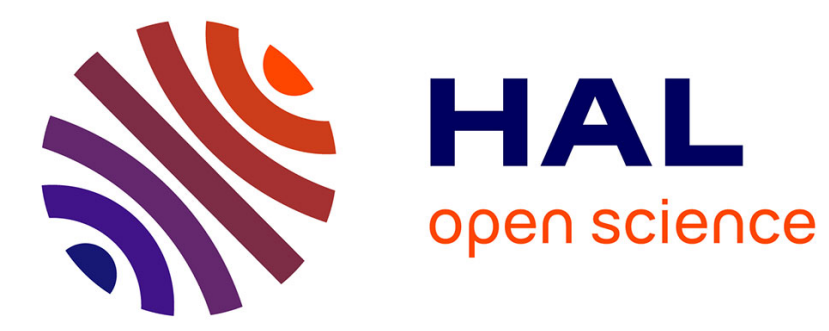

\title{
Human neutrophils swim and phagocytize bacteria
}

Nicolas Garcia-seyda, Valentine Seveau, Fabio Manca, Martine

Biarnes-pelicot, Marie-pierre Valignat, Marc Bajenoff, Olivier Theodoly

\section{To cite this version:}

Nicolas Garcia-seyda, Valentine Seveau, Fabio Manca, Martine Biarnes-pelicot, Marie-pierre Valignat, et al.. Human neutrophils swim and phagocytize bacteria. Biology of the Cell, 2020, 10.1111/boc. 202000084 . hal-02969578

\section{HAL Id: hal-02969578 https://hal.science/hal-02969578}

Submitted on 16 Oct 2020

HAL is a multi-disciplinary open access archive for the deposit and dissemination of scientific research documents, whether they are published or not. The documents may come from teaching and research institutions in France or abroad, or from public or private research centers.
L'archive ouverte pluridisciplinaire HAL, est destinée au dépôt et à la diffusion de documents scientifiques de niveau recherche, publiés ou non, émanant des établissements d'enseignement et de recherche français ou étrangers, des laboratoires publics ou privés. 
This is the peer reviewed version of the article, which has been published in its final form at [DOI: $10.1111 /$ boc.202000084]. This article may be used for non-commercial purposes in accordance with Wiley Terms and Conditions for Use of Self-Archived Versions. 


\section{Graphical abstract}

Bacterium phagocytosis in 3D

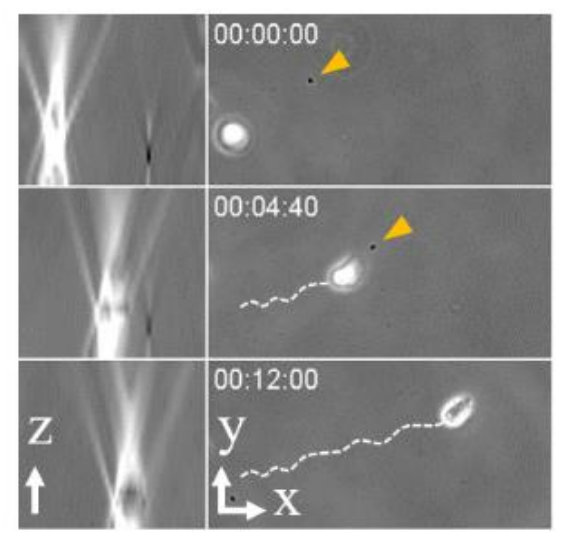

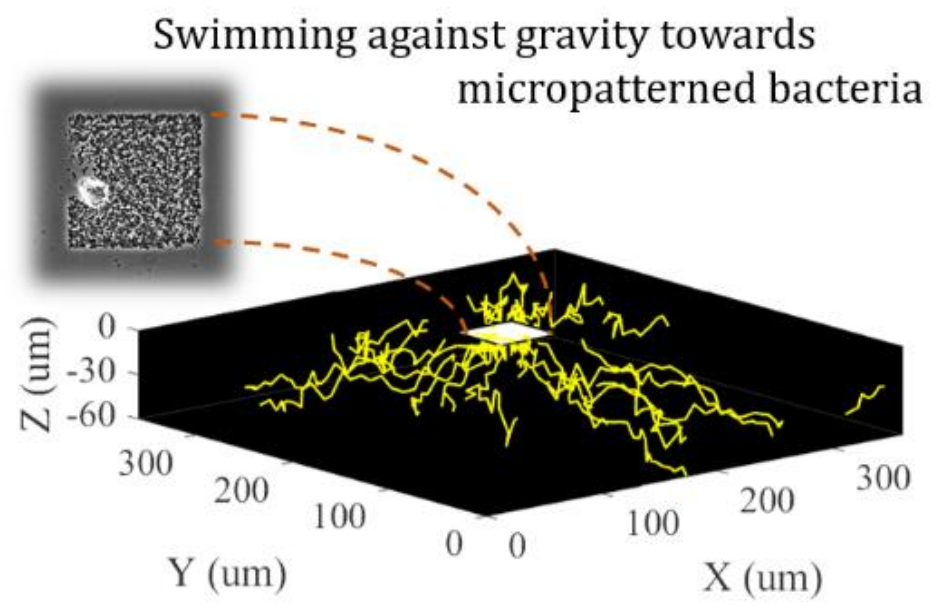

Leukocyte in vitro swimming was claimed twice within the last decade without discussing its physiological relevance, while clinical evidence shows leukocytes can be found in liquid-filled body cavities with phagocytized pathogens. Here we reproduced neutrophil swimming chemotaxis in 2 and 3D. Cells were capable of swimming against gravity towards a micropatterned spot of live bacteria; they furthermore performed phagocytosis while swimming, proving they do not rely on adhesion to carry out their functions. 


\title{
Human neutrophils swim and phagocytize bacteria
}

\author{
Nicolas Garcia-Seyda ${ }^{\mathrm{a}, \mathrm{b}}$, Valentine Seveau ${ }^{\mathrm{a}}$, Fabio Manca ${ }^{\mathrm{a}}$, Martine Biarnes-Pelicot ${ }^{\mathrm{a}}$, Marie-Pierre Valignat ${ }^{\mathrm{a}}$, Marc \\ Bajénoff $^{\mathrm{b}}$ and Olivier Theodoly ${ }^{\mathrm{a}^{*}}$ \\ ${ }^{a}$ Aix Marseille Univ, Inserm, CNRS, Turing Center for Living Systems, LAI, Marseille, France. \\ ${ }^{\mathrm{b}}$ Aix Marseille Univ, Inserm, CNRS, CIML, Marseille, France \\ *To whom correspondance should be addressed
}

\section{Statement of relevance}

We provide here evidence supporting the existence of leukocyte swimming, and we discuss its relevance in the light of recent in vivo findings. In particular, we verified a claim stated 10 years ago and never reproduced, on the capacity of human neutrophils to swim and perform swimming chemotaxis. We further extended those results to prove that swimming neutrophils can phagocytize bacteria, disregarding adhesion nor confinement as a requisite for accomplishing their function, which differs with current paradigms of leukocyte migration. From a biomedical aspect, we provide a tool to generate controlled, micron-size patterns of adhered, opsonized and live bacteria, applicable as a platform to study immune cell interactions, or their secretomes, upon pathogen-triggered activation.

\begin{abstract}
Leukocytes migrate in an amoeboid fashion while patrolling our organism in the search for infection or tissue damage. Their capacity to migrate has been proven integrin independent, however, non-specific adhesion or confinement remain a requisite in current models of cell migration. This idea has been challenged twice within the last decade with human neutrophils and effector $\mathrm{T}$ lymphocytes, which were shown to migrate in free suspension, a phenomenon termed swimming. While the relevance of leukocyte swimming in vivo remains under judgment, a growing amount of clinical evidence demonstrates that leukocytes are indeed found in liquid-filled body cavities, occasionally with phagocyted pathogens, such as in the amniotic fluid, the cerebrospinal fluid (CSF), or the eye vitreous and aqueous humor. Here, we studied in vitro swimming of neutrophils in the presence of live bacteria. We show that swimming neutrophils perform phagocytosis of bacteria in suspension, suggesting that they do not rely on adhesion to carry out their function. By micropatterning live bacteria on a substrate with an optical technique, we further prove that they use chemotaxis to swim towards their targets. Moreover, we provide evidence that neutrophil navigation can alternate between adherent and non-adherent modes, supporting a versatile phagocytic function adaptable to the various environmental conditions encountered in vivo.
\end{abstract}




\section{Introduction}

Leukocyte motility is an unquestioned requirement for the correct development of immune responses. Leukocytes migrate in a so-called 'ameboid' fashion, reminiscent of the shape deformations displayed by migrating Dictyostelium amoebae ${ }^{1}$. Amoeboid migration is generally defined by a fast displacement (typically between $5-20 \mu \mathrm{m} / \mathrm{min}$ ), lack of mature focal adhesions to the substrate, and lack of extracellular matrix degradation ${ }^{2}$. It is nevertheless widely assumed that mammalian cells require specific adhesion for crawling on unconfined 2D substrates ${ }^{3-7,7-11}$.

In 2010, Barry and Bretscher challenged this idea providing evidence that amoebae and human neutrophils swim in free suspension, suggesting rearward protrusion paddling or surface flow as the propelling engine ${ }^{12}$. While these initial observations were explained by others solely with protrusion paddling models ${ }^{13,14}$, the original authors did not support such claims, disregarding also surface flow as an alternative explanation ${ }^{15}$. Furthermore, as of today, swimming of human neutrophils has not been reproduced. Nonetheless, we recently reported that human Effector $\mathrm{T}$ lymphocytes also swim ${ }^{16}$. Supported by our experimental observations, we proposed a theoretical model for swimming migration based on a "molecular paddling", in which membrane components linked to actin couple the treadmilling cytoskeleton to the surrounding fluid, thereby propelling the cell forward. Swimming efficiency was further shown to be independent of medium viscosity and not influenced by the vicinity of a non-adherent 2D substrate. All in all, our model can explain amoeboid migration in various environmental conditions, namely $2 \mathrm{D}$ and $3 \mathrm{D}$, with or without adhesion, confined or in solution. Importantly, it also implies that all cells polarized in suspension and bearing a treadmilling cytoskeleton should swim.

A withstanding question though is whether leukocyte swimming occurs in vivo, and if it provides any functional advantage to the swimming cell. A growing number of clinical reports state that leukocytes, specially neutrophils, are found in liquid-filled body compartments, in some cases with ingested pathogens ${ }^{17-27}$. However, without live imaging it remains impossible to judge whether phagocytosis takes place in suspension or before detachment from the surrounding tissue.
Here, we reproduced in vitro swimming of primary human neutrophils polarized by the presence of live bacteria. We demonstrate that they use swimming chemotaxis to approach their target bacteria, and that phagocytosis takes place upon arrival. Furthermore, we provide evidence that neutrophils navigate on adherent substrates by alternating between adherent and non-adherent modes. Our data suggest these cells do not rely on adhesion to carry out their function, which may be of relevance during in vivo immune responses.

\section{Results}

We first recapitulated activation and swimming of neutrophils by seeding them on a non-adhesive substrate, passivated with Pluronic ${ }^{\circledR}$ F-127, in the presence of live Escherichia coli. While control neutrophils remained unpolarized, they quickly polarized and swam upon bacterial arrival (Fig 1A and movie 1). Swimming of neutrophils also occurred when they were incubated with $10 \mathrm{nM} \mathrm{N}$-formylmethionyl-leucyl-phenylalanine (fMLP) (Fig 1B and movie 2). Reflection Interference Contrast Microscopy (RICM) was used to assess lack of cell adhesion to F-127 or poly-ethylene glycol (PEG) treated substrates. With this imaging technique, cells in close contact to the substrate display destructive optical interference leading to a dark shade below their bodies, whereas non-adherent cells present constructive optical interference leading to a brighter intensity ${ }^{28}$. Expectedly, cells on either of the nonadhesive substrates presented bright signals (Fig 1C and movie 2). However, we also observed a fraction of non-adherent cells on adhesive ICAM-1 coated substrates (Fig 1C). Furthermore, cells migrated by alternating between high and low levels of adhesion (Fig 1D and movie 3).

When bacteria were opsonized with human serum and co-incubated with neutrophils on an adhesive substrate, phagocytosis took place (Fig 2A and movie 4). Moreover, when co-incubated in low numbers we could detect phagocytic events on non-adhesive substrates, with swimming neutrophils acting as scavengers to clear the field of view from bacteria (Fig 2B, 2C and movies 5 to 7). Bacteria phagocytosis was confirmed by incubation with $E$. coli bioparticles conjugated to the pHrodo $^{\mathrm{TM}}$ red sensor, which becomes fluorescent upon environment acidification within cell phagosomes. As expected from phagocyted 
bacteria, neutrophils increased their fluorescence during the live acquisition (Fig 2D and movie 8).

Phagocytic events could arise from random encounters between neutrophils and bacteria. However, the directed manner cells approached their targets suggested that swimming chemotaxis was taking place. We previously reported chemotaxis of adherent neutrophils towards live bacteria in a microfluidic device $^{29}$. Here, we micropatterned opsonized bacteria on a protein A coated spot within an otherwise nonadhesive PEG-coated substrate, to determine whether neutrophils follow chemotactic cues also while swimming. Such a target, fixed in space, was required in order to estimate cell directivity by scoring approach angles. Bacteria were viable, as accounted by their proliferation and escape from the micropatterned spot, and still able to activate neutrophils (Fig 3A and movie 9). To have the micropatterned spot as the only source of chemoattractants, we washed intensively the surrounding channel to get rid of any unbound bacteria and blocked their growth by supplementing the medium with Chloramphenicol. When neutrophils were seeded around, they displayed strong convergent chemotaxis towards the target (Fig 3B and movie 10). RICM imaging confirmed that while neutrophils adhered on the micropatterned area upon arrival, they approached it by swimming (Fig 3C and movie 11).

Such experiments proved 2D swimming chemotaxis for cells sedimented on a non-adherent substrate. To test 3D swimming chemotaxis, we repeated the experiment in medium supplemented with $15 \%$ High Molecular Weight Dextran. Such preparation increases medium density to almost match that of neutrophils ${ }^{30,31}$. More importantly it also increases viscosity, therefore slowing down cell sedimentation while swimming remains unaffected ${ }^{16}$. When the sample was flipped to have the micropatterned area on the upper side, neutrophils swam in 3D against gravity and towards the target (Fig 3D and Movie 12). In addition, using the same viscous medium we could record a phagocytic event in $3 \mathrm{D}$, for a bacterium drifting $10 \mu \mathrm{m}$ above the substrate (Fig 4 and Movie 13).

Based on our results, we conclude that human neutrophils constitutively migrate upon activation, either by adherent or swimming modes, while performing chemotaxis and carrying on with their phagocytic activity.
A

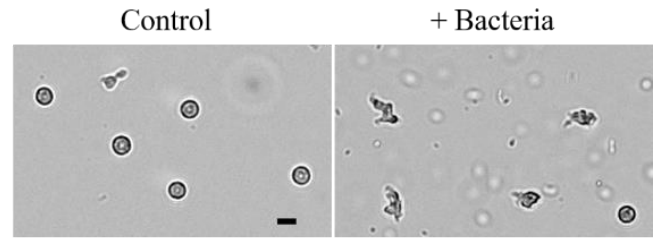

B

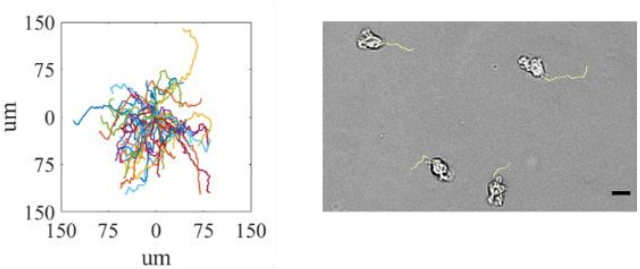

C

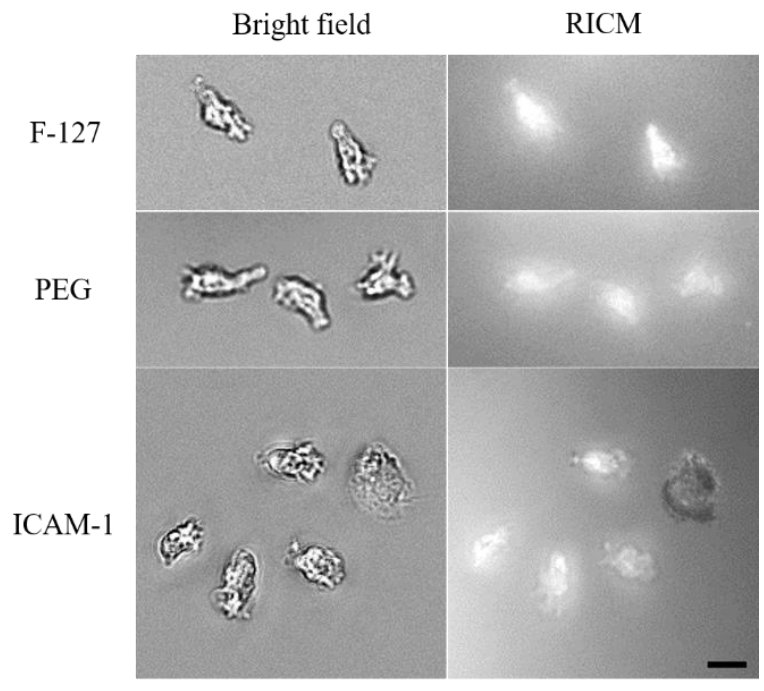

D

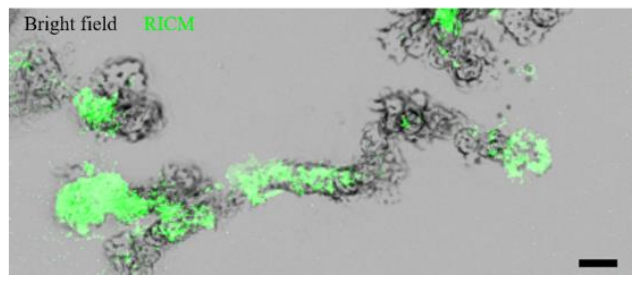

Fig 1 Neutrophils swim. (A) Control neutrophils alone or in the presence of live E. coli. on a F-127 substrate. Images are representative of 2 independent experiments. (B) Neutrophils swimming on a F-127 substrate in the presence of a homogeneous concentration of fMLP $(10 \mathrm{nM})$. Left, tracks aligned in the origin for 50 cells tracked over a $15 \mathrm{~min}$ interval. Right, snapshot of migrating neutrophils after a 2 min interval, with trailing tracks in yellow. Snapshot taken from movie 2, representative of 2 independent experiments. (C) Bright field (left) and raw RICM (right) images of migrating neutrophils on the indicated substrates. (D) Overlaid bright field (grey) and RICM (green = adhesion patch) projections, to reveal adhesion fingerprints while migrating on an ICAM-1 substrate. Image composite created from movie 3, representative of 3 independent experiments. Scalebars $=10 \mu \mathrm{m}$. 
A

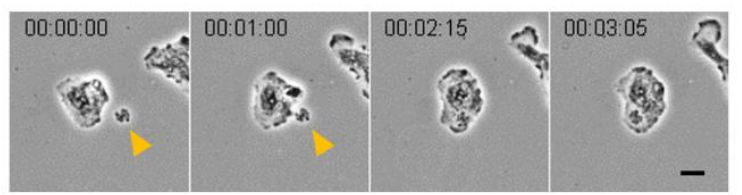

B

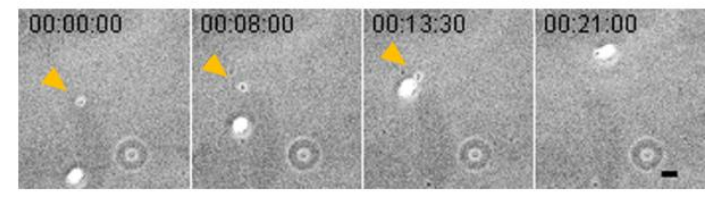

C
Remaining
4
3
2
1
0
bacteria:

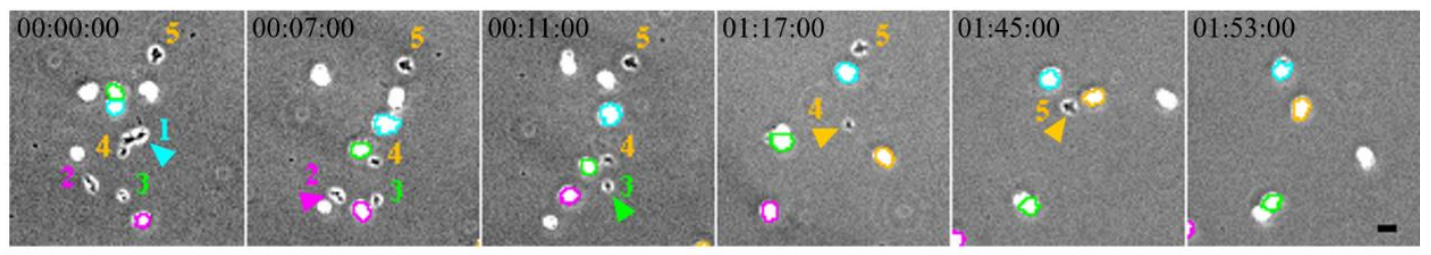

D

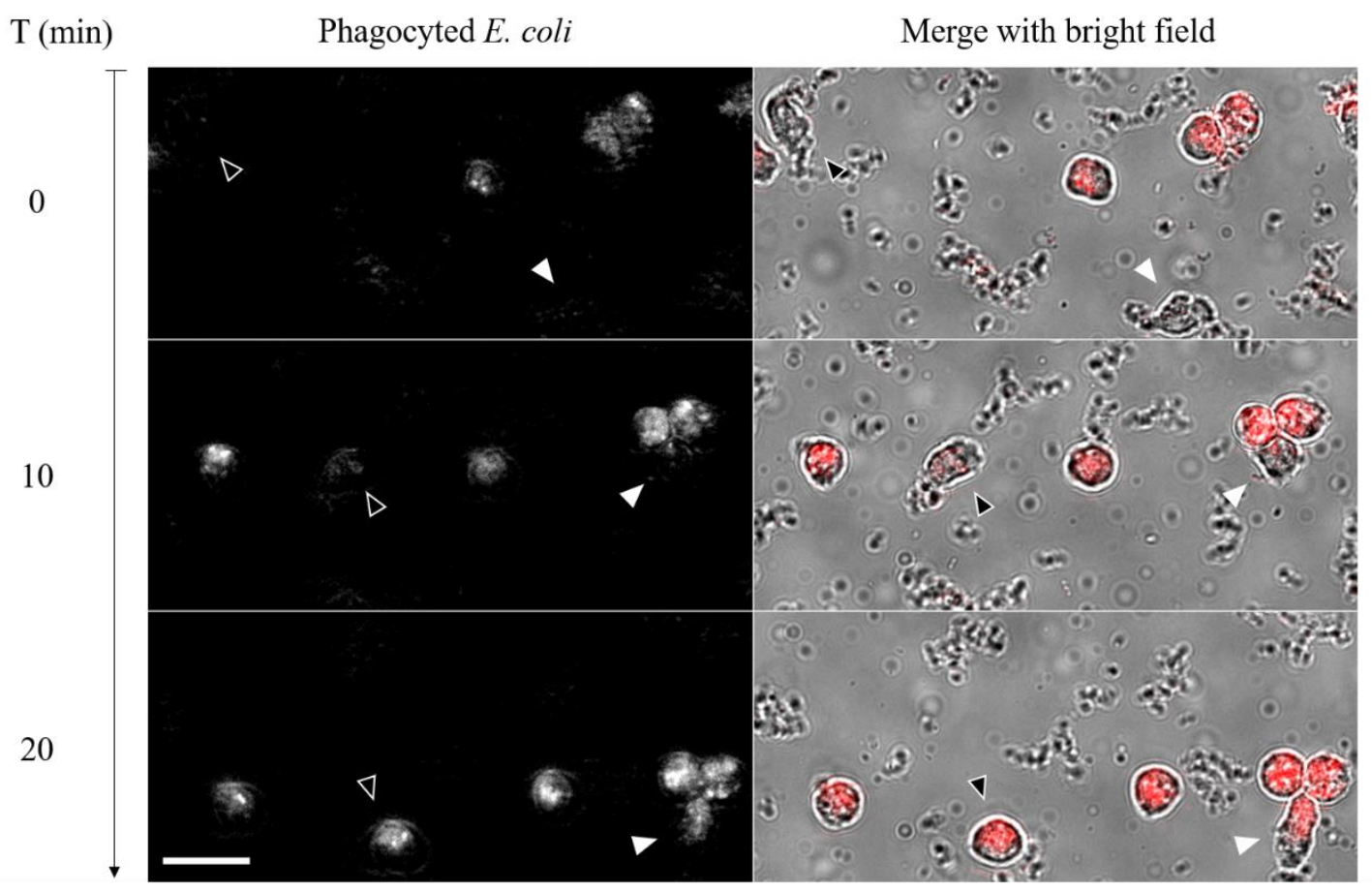

Fig 2 Neutrophils phagocytize while swimming. (A) Adherent neutrophil phagocyting sedimented bacteria debris, pointed out by the orange arrowhead. Scalebar $=10 \mu \mathrm{m}$. (B) Swimming neutrophil phagocyting a suspended bacterium, pointed out by the orange arrowhead. Montage created from movie 5. Scalebar $=20 \mu \mathrm{m}$. (C) Phase contrast imaging of swimming neutrophils scavenging bacteria over a $\sim 2 \mathrm{hs}$ period, until clearing the field of view. Neutrophils appear as white blobs whereas bacteria chunks appear dark. Each bacteria-neutrophil doublet is labeled with a distinctive color, arrowheads point at the bacterium about to be phagocyted. Bacteria 4 and 5 are phagocyted by the same neutrophil. Montage created from movie 7. Scalebar $=20 \mu \mathrm{m}$. (D) Swimming neutrophils phagocyting E. coli bioparticles conjugated to the pHrodo ${ }^{\mathrm{TM}}$ red sensor. Arrowheads point at two migrating cells whose fluorescence increases during the imaging period. Montage created from movie 8 . Scalebar $=20$ $\mu \mathrm{m}$. All time units are in the format hs:min:sec. 
B

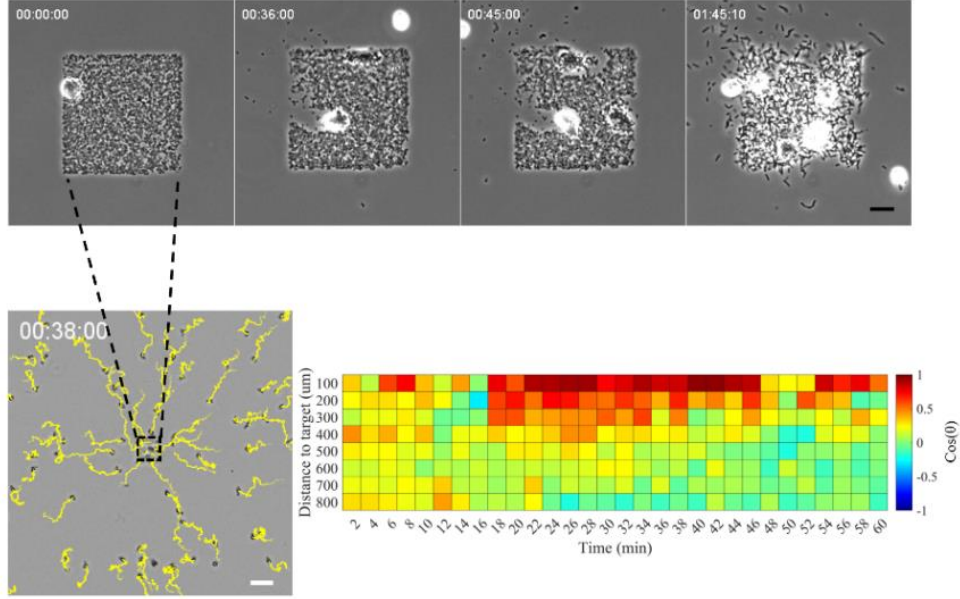

C

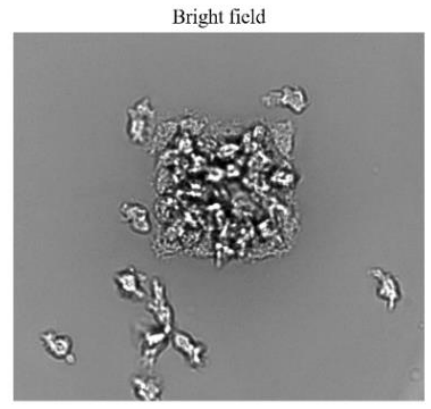

RICM

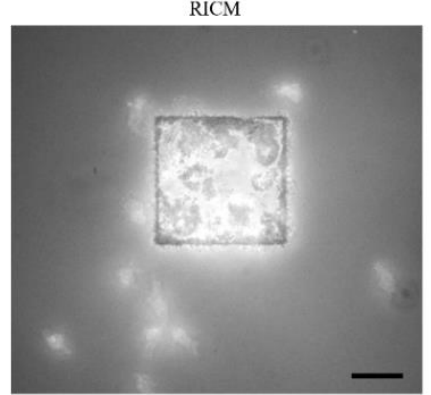

D
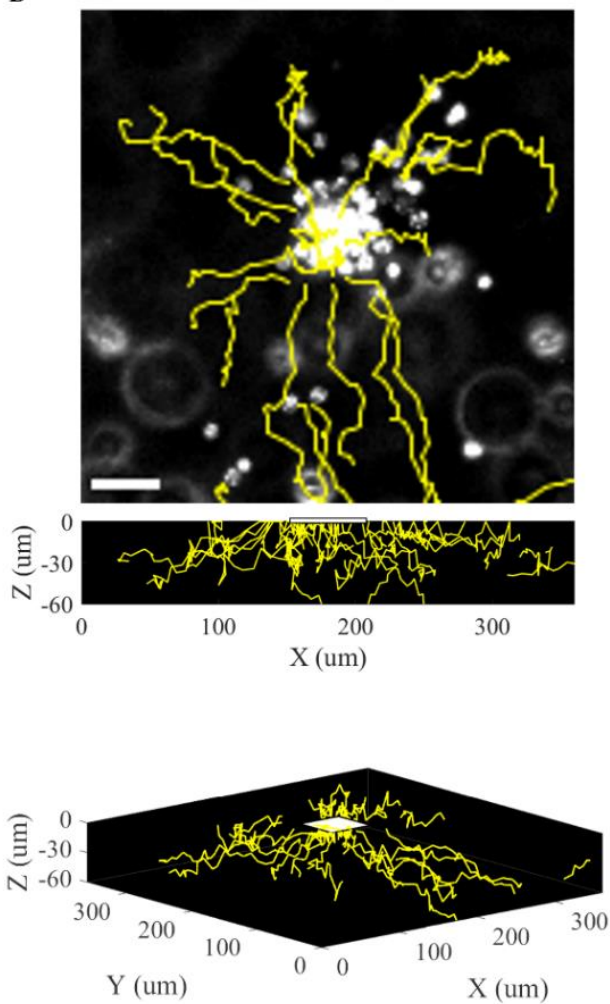

Fig 3 Neutrophils swimming in 2 and 3D perform chemotaxis to approach their targets. (A) Left, a carpet of micropatterned bacteria gets attacked by arriving neutrophils, while bacteria continue to proliferate and escape from the adhesive area. Montage created from movie 9. Scalebar $=20 \mu \mathrm{m}$. (B) Left, top-view snapshot of neutrophils swimming in 2D on a PEG-coated substrate, converging to their bacteria target. Trailing tracks are overlaid in yellow. Right, heatmap for the approaching angle as a function of time and radial distance to the target. Depicted is the $\cos (\theta)$, with $\theta$ being the angle between the straight line to the target at time $t$ and the cell's direction vector between $t$ and $t_{+1}$. A value of 1 represents directed radial migration towards the target, whereas a value of -1 represents directed radial escape from it. Values were calculated over 2 min intervals. Image and analysis created from movie 10, representative of 3 independent experiments. Scalebar $=50 \mu \mathrm{m}$. All time units are in the format hs:min:sec. (C) Bright field and RICM images of neutrophils arriving at the micropatterned area. Snaps taken from movie 11 . Scalebar $=20 \mu \mathrm{m}$. (D) Above, top-view snapshot of neutrophils swimming in 3D over a 2 hs period in medium supplemented with $15 \%$ High Molecular Weight Dextran, converging to their bacteria target. Trailing tracks are overlaid in yellow. Snap taken from movie 12. Scalebar $=50 \mu \mathrm{m}$. Below, side-view and $3 \mathrm{D}$ reconstructions to highlight upward motion towards the bacteria target, marked as a white square. 
Side view

$(\mathrm{x}, \mathrm{z})$
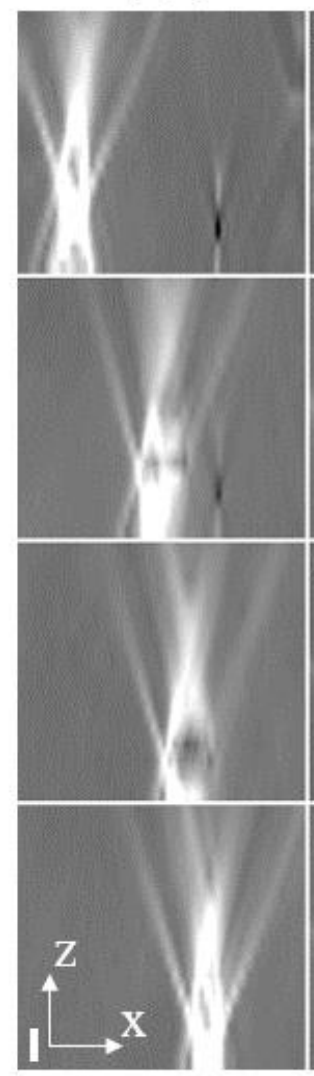

Top view

(x, y, z=10 $10 \mathrm{~m}$ )
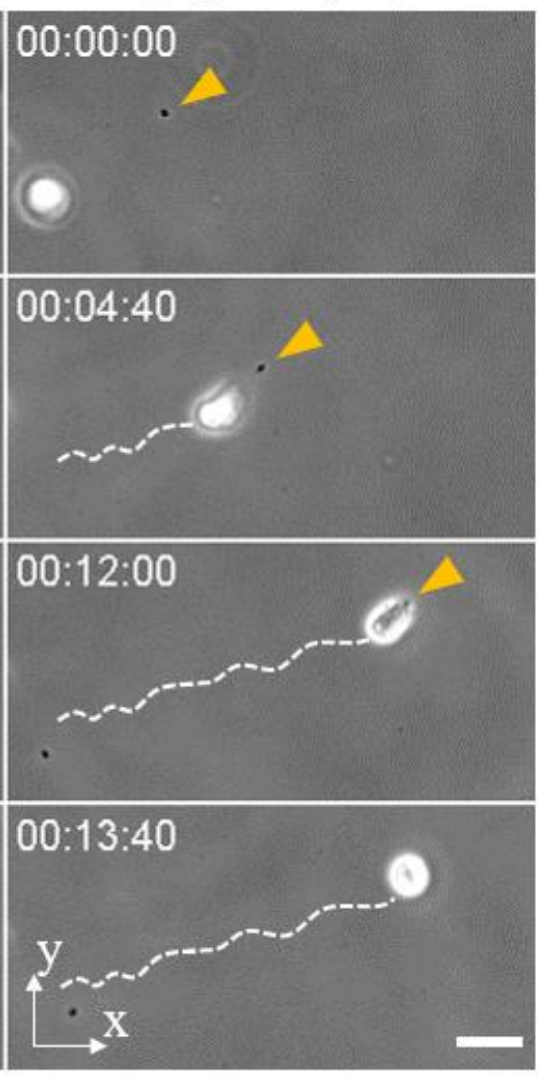

Focus plane

(z)

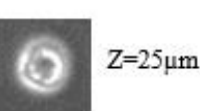

$D_{x, y}=38 \mu \mathrm{m}$

- $\quad Z=10 \mu \mathrm{m}$

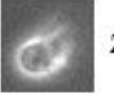

$Z=15 \mu \mathrm{m}$

$D_{s, y}=10 \mu m$

1. $\quad Z=10 \mu \mathrm{m}$

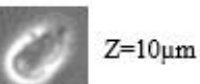

$\mathrm{D}_{\mathrm{x}, \mathrm{y}}=1 \mu \mathrm{m}$

. $Z=10 \mu \mathrm{m}$

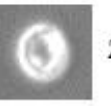

$Z=10 \mu \mathrm{m}$

$D_{x, y}=0 \mu \mathrm{m}$

Distance

$(\mathrm{x}, \mathrm{y})$$$
\text { Dive }
$$

$\approx$

Fig 4 Phagocytosis also occurs during swimming in 3D. Left, side and top views of a phagocytic event occurring $10 \mu \mathrm{m}$ above the substrate. The side view is performed along the shortest straight line between the cell and the bacteria. The orange arrowhead points to the bacterium position. Right, cropped in-focus planes of the cell and the bacteria for each timepoint, and the linear $(\mathrm{x}, \mathrm{y})$ distance separating them. Snaps taken from movie 13. Scalebar $(\mathrm{x}, \mathrm{z})=$ $10 \mu \mathrm{m}$, scalebar $(\mathrm{x}, \mathrm{y})=20 \mu \mathrm{m}$. 


\section{Discussion}

Leukocyte amoeboid migration has been studied in a variety of experimental conditions, though almost always in the presence of adhesion, confinement, or both. Therefore, the capacity of leukocytes to perform swimming, that is the migration in the absence of confinement or adhesion, has remained controversial ever since it was described. Here, 10 years after its initial and unique report ${ }^{12}$, we have reproduced swimming of primary human neutrophils, recording swimming trajectories in 3D and against gravity. Our data show that these cells, upon activation by fMLP or bacterial secreted compounds, likely Pathogen Associated Molecular Patterns (PAMPs), trigger a constitutively active migration mechanism to approach their targets. If an adherent substrate is available, they adhere, otherwise they continue swimming. The observed swimming chemotaxis towards bacteria strongly resembles the clustering dynamics of mouse neutrophils in vivo swarming around laser-induced wounds ${ }^{32}$, and human neutrophils in vitro swarming around zymosan particles ${ }^{33}$. Interestingly, in the latter report no adhesion molecule was grafted in between the arrays of zymosan particles, suggesting that the recorded migration was sustained either by non-specific adhesion or swimming.

Neutrophil interstitial migration towards the inflammation site was proven integrin independent, though under tissue confinement ${ }^{32}$. Here, we extend this observation to unconfined swimming, which may be especially important upon reaching an infection site. During inflammation, the release of tissue degrading enzymes such as matrix metalloproteases (MMPs) by the pathogen and host macrophages leads to extracellular matrix (ECM) breakdown and loss of tissue integrity ${ }^{34,35}$. Activated neutrophils themselves secrete a variety of proteases (such as collagenase, neutrophil elastase and metalloproteases) and Reactive Oxygen Species that could also damage the tissue matrix at the infection site ${ }^{36,37}$. Swimming could therefore provide a mechanism allowing cells to continue their march to the very frontline, regardless the potential lack of adhesion or confinement. We identify edema, skin pimples and blisters as potential sites where swimming would be relevant upon tissue swelling or pus secretion. There are additional environments in the organism that remain permanently filled with liquid and where pathogens and leukocytes, principally neutrophils, have been detected. These are the cerebrospinal fluid (CSF) ${ }^{17-}$ ${ }^{21}$, the eye vitreous and aqueous humor ${ }^{22-24}$, and the amniotic fluid $^{25-27}$. For the latter case, amniotic neutrophils where found with a variety of ingested pathogenic bacteria, and the ex-vivo phagocytosis of the same pathogens was recorded ${ }^{25}$. While those tests were done under adherent conditions, neutrophil phagocytic activity has also been reported while in an Eppendorf tube, likely representing swimming phagocytosis ${ }^{25,38-40}$.

If any of the above-mentioned tissues suffers from infection, swimming leukocytes are required to clear out the pathogen, since a single bacterium remaining in the bulk could act as a reservoir leading to chronic infection. Leukocytes will therefore need to quit adhesion and swim in order to reach it, a behavior already described for effector $\mathrm{T}$ lymphocytes ${ }^{16}$. In line with this, we provide here evidence that neutrophils can migrate on adherent substrates by alternating between high and low adhesion levels. An additional point is that eukaryotic cells sediment faster than bacteria, therefore quitting adhesion would not be enough per se, as leukocytes would quickly sediment. Indeed, reported experiments with swimming cells were done in medium supplemented with Ficoll, to avoid cell sedimentation ${ }^{12,13,16,41}$. However, it has been reported that activated neutrophils from septic samples display an increased volume, which decreases their density and therefore prevents their sedimentation in the blood column, a property measured as Leukocyte Antisedimentation Rate (LAR) $)^{42,43}$. As shown in Fig 3D and Movie 12, neutrophils can indeed swim against gravity if the medium is dense or viscous enough. Finally, while canceling residual drifts proves essential to study cell swimming in vitro, the in vivo situation is unlikely to be flow-free. We envision a scenario where cells simply detach from the surrounding tissue and are taken by the fluid drift. While fluid mixing abrogates the establishment of long-distance gradients, diffusion is instead dominant at short scales. Therefore, directed swimming taking place in the proximity of bacteria would help neutrophils to reach out and capture targets, a similar landscape to the one described for marine microbes ${ }^{44}$. For 'blind' leukocytes, extending a protrusion in the right direction would be the sole way of capturing a bacterium drifting few microns away, as illustrated by Movie 13 .

Furthermore, while other models explain amoeboid migration only in confining substrates ${ }^{9,11,45-48}$, our model also explains migration in solution. Regardless the mechanism, cells are motile in a wide range of environments. This would explain the capacity of immune cells to keep immunosurveillance in all body tissues. It is worth to note though, that so far only 2 primary cell types from higher eukaryotes have been reported to swim: 
neutrophils and effector T lymphocytes, both of human origin. Perhaps leukocyte swimming is a unique feature of human cells, however, if amoeboid migration is an inherited ancient mechanism it should be preserved across different species. This is especially true in the light of our model, which implies that any polarized cell with a treadmilling cytoskeleton should be able to swim. Future tests with animal models will determine whether swimming of leukocytes takes place in vivo, and if it is species-preserved.

As a final remark, our method for live bacteria micropatterning can be readily applied as microarrays for biomedical applications like those reported by Reátegui et al. ${ }^{33}$ using zymosan particles, to investigate neutrophil secreted compounds during chemotaxis and swarming, but towards a live pathogen ${ }^{49}$. Moreover, our images of neutrophils 'hoovering' a bacteria carpet (Fig 3A and movie 9) strongly resemble that of bacteria scavenging by platelets, as recently reported ${ }^{50}$. It will be interesting to perform co-incubation experiments with those and other immune cell actors, to mimic the early steps of the immune response and reach an Infection-on-a-Chip model.

\section{Author contributions}

NGS and OT designed the experiments, VS prepared the substrates for bacteria micropatterning, NGS and FM wrote the scripts for analysis of chemotaxis analysis, NGS performed the experiments, analyzed the data and wrote the manuscript, MPV, MB, MBP and OT revised it. OT supervised the project.

\section{Acknowledgements}

This work was supported by Agence Nationale de la Recherche (RECRUTE - ANR-15-CE15-0022 and ILIAAD ANR-18-CE09-0029); LABEX INFORM; and the Turing Centre for Living systems. This project has received funding from the European Union's Horizon 2020 research and innovation programme under the Marie Skłodowska-Curie grant agreement No713750. Also, it has been carried out with the financial support of the Regional Council of Région Sud and with the financial support of Excellence Initiative of Aix-Marseille University $-\mathrm{A}^{*}$ MIDEX, a French "Investissements d'Avenir" " project funded by the French Government, managed by the French National Research Agency (ANR). We are also grateful to the Cell Culture Platform facility (Luminy TPR2-INSERM).

\section{Methods}

\section{Cells}

Whole blood from healthy adult donors was obtained from the Établissement Français du Sang. Neutrophils were extracted within 2 hs after drawn with the EasySep ${ }^{\mathrm{TM}}$ Direct Human Isolation Kit (STEMCELL Technologies), following manufacturer instructions. After purification, cells were resuspended in RPMI 1640 medium supplemented with penicillin $100 \mathrm{U} / \mathrm{mL}$ (Gibco, Carlsbad, CA), streptomycin $100 \mu \mathrm{g} / \mathrm{mL}$ (Gibco, Carlsbad, CA), $25 \mathrm{mM}$ GlutaMax (Gibco, Carlsbad, CA), and 10\% fetal calf serum (FCS; Lonza, Basel, Switzerland), and used right away.

\section{Bacteria preparation}

Escherichia coli (DH5a strain) cultures were grown overnight in LB medium (Invitrogen), pH 7. The next day cells were washed once in Phosphate Buffer Solution (PBS) (Gibco), pH 7.4, resuspended in Human male AB Serum (Sigma Aldrich) and incubated at $37^{\circ} \mathrm{C}$ with agitation for at least $1 \mathrm{hr}$. Alternatively, pHrodo ${ }^{\mathrm{TM}} \operatorname{Red} E$. coli bioparticles (ThermoFisher) were used. Subsequently, opsonized cells were washed 3 times with PBS and directly used for coincubation experiments or micropatterning. Similar results were obtained when heat inactivated serum was used.

\section{Substrate preparation}

Microfluidic channels (Ibidi Slide IV 0.4) were coated with $10 \mu \mathrm{g} / \mathrm{mL}$ human ICAM-1-Fc (R\&D Systems) overnight at $4{ }^{\circ} \mathrm{C}$. Channels were subsequently blocked with $4 \%$ bovine serum album (BSA) for at least $15 \mathrm{~min}$ at room temperature, and rinsed with PBS and RPMI medium prior to use. Non-adherent substrates were prepared either by incubating the channels with 5\% Pluronic F-127 (Sigma-Aldrich) for at least $30 \mathrm{~min}$ at room temperature, or by mPEG SVA treatment following the same protocol as described for bacteria micropatterning.

\section{Bacteria micropatterning}

Glass slides (Schott High performance coverslip $75 \times 25 \# 1.5 \mathrm{H}$ cleanroom cleaned) were plasma activated (Harrick Plasma) during 5min, then a 1\% APTES 0.03\% Acetic acid solution was poured on them and incubated for 2 hours at $4^{\circ} \mathrm{C}$. Subsequently slides were rinsed with Milli-Q water, dried and baked $15 \mathrm{~min}$ at $95^{\circ} \mathrm{C}$, finally microfluidic channels were built by sticking an Ibidi 
Sticky-Slide VI 0.4 on top. A solution of $23 \%$ mPEG SVA (MW:5000 Da, INTERCHIM) 10\% NaHCO3 was introduced into the channels and devices were incubated overnight at $4^{\circ} \mathrm{C}$. The next day channels were rinsed with PBS and blocked for 15 min with 4\% BSA. Excess of liquid was removed from the wells, PLPP solution (Alvéole) was added into the channels and a $250 \mu \mathrm{L}$ glass syringe filled with $100 \mu \mathrm{L}$ PLPP was plugged to the device. The syringe was installed on a pump (neMESYS $290 \mathrm{~N}$, Cetoni) with an alternating flow of $+/-12 \mu \mathrm{L} / \mathrm{sec}$ and controlled by neMESYS user interface. Patterns of interest were designed on Matlab (The MathWorks, Natick, MA, USA) and exposed on the device with UV $\left(\lambda=375 \mathrm{~nm}\right.$ for $160 \mathrm{sec}, \sim 2212 \mathrm{~mJ} / \mathrm{mm}^{2}$ dose) through a Digital Micromirror Device (Primo ${ }^{\mathrm{TM}}$, Alvéole). Channels were rinsed with PBS, and a $50 \mu \mathrm{g} / \mathrm{mL}$ solution of protein A (AF647 conjugated, Invitrogen) was incubated during $45 \mathrm{~min}$ at room temperature. Channels were rinsed with PBS and blocked for 15 min with $4 \%$ BSA. After a final rinsing with PBS, opsonized bacteria were introduced in the channels and the whole device was centrifuged for $3 \mathrm{~min}$ at $1000 \mathrm{RCF}$. Unbound bacteria were removed with intense PBS rinsing, and the device was kept at $4^{\circ} \mathrm{C}$ until use. Before starting the experiment, a final rinse was performed with cold RPMI 1640 medium to remove bacterial chemoattractants, then neutrophils were added, and the acquisition was immediately launched. For 3D experiments, neutrophils were resuspended in RPMI medium supplemented with $15 \%$ Dextran (1500-2800 kDa). In order to flip over the sample, the micropattern was done in an open well which was then inverted on two spacers glued to a petri dish, to create a channel without medium leakage due to gravity. The petri dish was finally filled with medium to abrogate flow in the channel.

\section{Imaging and data analysis}

Experiments were performed on an inverted Zeiss Z1 automated microscope (Carl Zeiss, Germany) equipped with a CoolSnap HQ CCD camera (Photometrics) and piloted by $\mu$ Manager1.4. A Neofluar 63/1.25 antiflex was used for Reflection Interference Contrast Microscopy (RICM) mode, in combination with a narrow band-pass filter $(\lambda=546 \mathrm{~nm} \pm 12 \mathrm{~nm}$ ). Images were taken every 10 sec intervals for $2 \mathrm{D}$ experiments and $60 \mathrm{sec}$ for 3D phase contrast imaging. Cells were tracked using the FIJI plugin Trackmate ${ }^{51}$. Tracks were exported and further analysis and plots were performed with MATLAB custom-made scripts. For the approach angle analysis, the first $50 \mu \mathrm{m}$ around the target were discarded to account only for swimming cells.

\section{Ethics statement}

Human subjects: Blood from healthy volunteers was obtained through a formalized agreement with French Blood Agency (Etablissement Français du Sang, agreement $\mathrm{n}^{\circ}$ 2017-7222). Blood was obtained by the agency after informed consent of the donors, in accordance with the Declaration of Helsinki. All experiments were approved by the INSERM Institutional Review Board and ethics committee. 


\section{References}

1. Friedl, P. \& Weigelin, B. Interstitial leukocyte migration and immune function. Nature immunology 9, 960-9 (2008).

2. Wolf, K., Müller, R., Borgmann, S., Bröcker, E.-B. \& Friedl, P. Amoeboid shape change and contact guidance: T-lymphocyte crawling through fibrillar collagen is independent of matrix remodeling by MMPs and other proteases. Blood 102, 3262-3269 (2003).

3. Bergert, M. et al. Force transmission during adhesion-independent migration. Nature Cell Biology 17, 524-529 (2015).

4. Nourshargh, S., Hordijk, P. L. \& Sixt, M. Breaching multiple barriers: leukocyte motility through venular walls and the interstitium. Nat. Rev. Mol. Cell Biol. 11, 366-378 (2010).

5. Bergert, M., Chandradoss, S. D., Desai, R. A. \& Paluch, E. Cell mechanics control rapid transitions between blebs and lamellipodia during migration. PNAS 109, 14434-14439 (2012).

6. Jacobelli, J., Bennett, F. C., Pandurangi, P., Tooley, A. J. \& Krummel, M. F. Myosin-IIA and ICAM-1 Regulate the Interchange between Two Distinct Modes of T Cell Migration. The Journal of Immunology 182, 2041-2050 (2009).

7. Smith, A. et al. A talin-dependent LFA-1 focal zone is formed by rapidly migrating $\mathrm{T}$ lymphocytes. The Journal of Cell Biology 170, 141 (2005).

8. Friedl, P. \& Wolf, K. Plasticity of cell migration: a multiscale tuning model. J Cell Biol 188, $11-19$ (2010).

9. Ruprecht, V. et al. Cortical Contractility Triggers a Stochastic Switch to Fast Amoeboid Cell Motility. Cell 160, 673-685 (2015). 
10. Renkawitz, J. \& Sixt, M. Mechanisms of force generation and force transmission during interstitial leukocyte migration. EMBO reports $\mathbf{1 1}, 744-750$ (2010).

11. Reversat, A. et al. Cellular locomotion using environmental topography. Nature 582, 582585 (2020).

12. Barry, N. P. \& Bretscher, M. S. Dictyostelium amoebae and neutrophils can swim. Proceedings of the National Academy of Sciences 107, 11376-11380 (2010).

13. Haastert, P. J. M. V. Amoeboid Cells Use Protrusions for Walking, Gliding and Swimming. PLOS ONE 6, e27532 (2011).

14. Bae, A. J. \& Bodenschatz, E. On the swimming of Dictyostelium amoebae. Proc. Natl. Acad. Sci. U.S.A. 107, E165-166 (2010).

15. Howe, J. D., Barry, N. P. \& Bretscher, M. S. How Do Amoebae Swim and Crawl? PLoS ONE 8, e74382 (2013).

16. Aoun, L. et al. Amoeboid Swimming Is Propelled by Molecular Paddling in Lymphocytes. Biophysical Journal (2020) doi:10.1016/j.bpj.2020.07.033.

17. Dietzel, J., Krebs, A., Lüdemann, J., Roser, M. \& Dressel, A. Neutrophils in cerebrospinal fluid without pleocytosis. European journal of neurology: the official journal of the European Federation of Neurological Societies 15, 634-6 (2008).

18. Schmitt, C., Strazielle, N. \& Ghersi-Egea, J.-F. Brain leukocyte infiltration initiated by peripheral inflammation or experimental autoimmune encephalomyelitis occurs through pathways connected to the CSF-filled compartments of the forebrain and midbrain. Journal of Neuroinflammation 9, 187 (2012).

19. Liu, Y.-W., Li, S. \& Dai, S.-S. Neutrophils in traumatic brain injury (TBI): friend or foe? Journal of Neuroinflammation 15, 146 (2018). 
20. Quagliarello, V. J., Long, W. J. \& Scheld, W. M. Morphologic alterations of the blood-brain barrier with experimental meningitis in the rat. Temporal sequence and role of encapsulation. J Clin Invest 77, 1084-1095 (1986).

21. Del Maschio, A. et al. Leukocyte Recruitment in the Cerebrospinal Fluid of Mice with Experimental Meningitis Is Inhibited by an Antibody to Junctional Adhesion Molecule (Jam). J Exp Med 190, 1351-1356 (1999).

22. Mashimo, H. et al. Neutrophil Chemotaxis and Local Expression of Interleukin-10 in the Tolerance of Endotoxin-Induced Uveitis. Invest. Ophthalmol. Vis. Sci. 49, 5450-5457 (2008).

23. Liu, K., Klintworth, G. K. \& Dodd, L. G. Cytologic findings in vitreous fluids. Analysis of 74 specimens. Acta Cytol. 43, 201-206 (1999).

24. Jiang, Z.-X. et al. Roscovitine ameliorates endotoxin-induced uveitis through neutrophil apoptosis. Mol Med Rep 14, 1083-1090 (2016).

25. Gomez-Lopez, N. et al. Amniotic Fluid Neutrophils Can Phagocytize Bacteria: A Mechanism for Microbial Killing in the Amniotic Cavity. Am J Reprod Immunol 78, (2017).

26. Gomez-Lopez, N. et al. Neutrophil Extracellular Traps in the Amniotic Cavity of Women with Intra-Amniotic Infection: A New Mechanism of Host Defense. Reprod Sci 24, 11391153 (2017).

27. Romero, R. et al. Neutrophil attractant/activating peptide-1/interleukin-8 in term and preterm parturition. Am. J. Obstet. Gynecol. 165, 813-820 (1991).

28. Theodoly, O., Huang, Z.-H. \& Valignat, M.-P. New Modeling of Reflection Interference Contrast Microscopy Including Polarization and Numerical Aperture Effects: Application to 
Nanometric Distance Measurements and Object Profile Reconstruction. Langmuir 26, 19401948 (2010).

29. Garcia-Seyda, N. et al. Microfluidic device to study flow-free chemotaxis of swimming cells. Lab Chip (2020) doi:10.1039/D0LC00045K.

30. Mach, O. \& Lacko, L. Density gradient in a dextran medium. Analytical Biochemistry 22, 393-397 (1968).

31. Zipursky, A., Bow, E., Seshadri, R. S. \& Brown, E. J. Leukocyte density and volume in normal subjects and in patients with acute lymphoblastic leukemia. Blood 48, 361-371 (1976).

32. Laemmermann, T. et al. Neutrophil swarms require LTB4 and integrins at sites of cell death in vivo. Nature 498, 371-+ (2013).

33. Reátegui, E. et al. Microscale arrays for the profiling of start and stop signals coordinating human-neutrophil swarming. Nat Biomed Eng 1, 0094 (2017).

34. Tomlin, H. \& Piccinini, A. M. A complex interplay between the extracellular matrix and the innate immune response to microbial pathogens. Immunology 155, 186-201 (2018).

35. Boyd, D. F. \& Thomas, P. G. Towards integrating extracellular matrix and immunological pathways. Cytokine 98, 79-86 (2017).

36. Padmanabhan, J. \& Gonzalez, A. L. The effects of extracellular matrix proteins on neutrophil-endothelial interaction--a roadway to multiple therapeutic opportunities. Yale $J$ Biol Med 85, 167-185 (2012).

37. Witko-Sarsat, V., Rieu, P., Descamps-Latscha, B., Lesavre, P. \& Halbwachs-Mecarelli, L. Neutrophils: Molecules, Functions and Pathophysiological Aspects. Laboratory Investigation 80, 617-653 (2000). 
38. Fleischmann, J., Golde, D. W., Weisbart, R. H. \& Gasson, J. C. Granulocyte-macrophage colony-stimulating factor enhances phagocytosis of bacteria by human neutrophils. Blood $\mathbf{6 8}$, $708-711(1986)$.

39. Bredius, R. G. M. Role of neutrophil Fc-yRIIa (CD32) and Fc-yRIIIb (CD16) polymorphic forms in phagocytosis of human IgG1- and IgG3-opsonized bacteria and erythrocytes. 7.

40. Sanders, L. A. M. et al. Human Immunoglobulin G (IgG) Fc Receptor IIA (CD32)

Polymorphism and IgG2-Mediated Bacterial Phagocytosis by Neutrophils. INFECT. IMMUN. 63, 9 (1995).

41. O’Neill, P. R. et al. Membrane Flow Drives an Adhesion-Independent Amoeboid Cell Migration Mode. Developmental Cell 46, 9-22.e4 (2018).

42. Bogar, L., Tarsoly, P. \& Jakso, P. Characteristics of light and heavy polymorphonuclear leukocytes. Clin. Hemorheol. Microcirc. 27, 149-153 (2002).

43. Bogar, L. Sedimentation characteristics of leucocytes can predict bacteraemia in critical care patients. Journal of Clinical Pathology 59, 523-525 (2006).

44. Stocker, R. Marine Microbes See a Sea of Gradients. Science 338, 628-633 (2012).

45. Bergert, M. et al. Force transmission during adhesion-independent migration. Nature Cell Biology 17, 524-529 (2015).

46. Hawkins, R. J. et al. Pushing off the Walls: A Mechanism of Cell Motility in Confinement. Physical Review Letters 102, (2009).

47. Tozluoğlu, M. et al. Matrix geometry determines optimal cancer cell migration strategy and modulates response to interventions. Nature Cell Biology 15, 751-762 (2013).

48. Wilson, K. et al. Mechanisms of leading edge protrusion in interstitial migration. Nature Communications 4, (2013). 
49. Lämmermann, T. Cell migration: Arraying neutrophils in swarms. Nat Biomed Eng 1, 0100 (2017).

50. Gaertner, F. et al. Migrating Platelets Are Mechano-scavengers that Collect and Bundle Bacteria. Cell 171, 1368-1382.e23 (2017).

51. Tinevez, J.-Y. et al. TrackMate: An open and extensible platform for single-particle tracking. Methods 115, 80-90 (2017). 